\title{
A study on OpenFOAM's Overset Mesh Support using flow past NACA 0018 Airfoil
}

\author{
Praveen Laws ${ }^{1}$, Jaskaran Singh Saini², Ajit Kumar ${ }^{3}$ \\ ${ }^{1}$ School of Engineering, Department of Mechanical Engineering, Shiv Nadar University, \\ Tehsil Dadri 203207, India \\ ${ }^{2}$ School of Mechanical, Industrial and Manufacturing Engineering, Oregon State University, \\ Corvallis, OR 97331, USA \\ ${ }^{3}$ School of Natural Sciences, Department of Mathematics, Shiv Nadar University, Tehsil \\ Dadri 203207, India
}

\begin{abstract}
The open source CFD code OpenFOAM has emerged as one of the most popular alternative to commercial CFD solvers. The recent version of OpenFOAM supports overlapping grids, so called Overset mesh. In this type of mesh, one or more sub-collection of control volumes (or cells) are allowed to overlap with other set of cells. This allows for great flexibility in modelling Fluid Structure Interaction (FSI) problems. Fluid mesh can be standard structured mesh of a rectangular/cuboidal/or cylindrical domain. The structure mesh can be generated separately by creating multiple layers around a given body. The overset mesh functionality allows for overlapping or immersing this structured mesh inside the fluid mesh and a relevant FSI or moving dynamics problems can be solved. The idea of overset mesh has been around since eighties but its support in OpenFOAM is very recent. Most CFD codes which support overset mesh have either been in-house or commercial CFD codes. OpenFOAM's support for overset is the first major open source code resource available for CFD problems. The aim of this research is to solve a classical benchmark airfoil problem using OpenFOAM overset mesh and compare the numerical results with experimental result. We report here flow simulations results around NACA 0018.The result obtained from overset mesh compares convincingly well with experimental results. Computations have been carried out for Reynolds numbers in the range of $10^{5}$ with angle of attack ranging from $\alpha=5^{\circ}$ to $\alpha=30^{\circ}$ with an interval of $5^{\circ}$. Turbulence is incorporated using $k-\varepsilon$ turbulence model. This study helps developing confidence in using OpenFOAM overset support for more complicated flows and moving dynamics. This report is complemented with a brief description of finite volume discretization using overset mesh.
\end{abstract}

Keywords: OpenFOAM, Overset Meshing, NACA 0018, High Reynolds numbers, CFD.

\section{Introduction}

Computational Fluid Dynamics (CFD) is now one of the routine tool for engineers, predominantly in the field of aerodynamics, hydrodynamics and automobile industry. Predictions of lift, drags, and other force components on a static body is a methodical calculation in any CFD simulation. However, flow prediction with moving boundaries like propellers, turbines, flexible bodies and complex structures still remains challenging. These fall under the purview of Fluid-Structure Interactions (FSI) which involves simultaneous solution of two sets of partial differential equations, one for fluid flow and calculation of pressure and stress, and another set of equation for structural deformation and dynamics. The

Corresponding Author: Dr. Ajit Kumar

Email: ajit.kumar@snu.edu.in 
coupling between fluid and structure equations are complicated but what is even more complicated is to adjust the mesh to incorporate the structure deformation. Typically, a structure is treated as a boundary for fluid flow and as soon as the structure is allowed to move, the mesh quality of the fluid flow gets compromised. Several techniques have been invented to allow for structure motion inside a fluid domain like the Immersed Boundary Method (IBM), Volume-of-Fluid method (VoF) and Level-Set method. The classical IBM is a mixed Eulerian and Lagrangian technique where the fluid is discretized by Cartesian grid while the solid by Lagrangian mesh. The solid mesh is free to move over the fluid Cartesian grid independently. The communication between the two different grids are performed by the smooth Dirac Delta function [1] [2]. The original IBM by Peskin was developed for FSI in biological flows, which cannot handle rigid body interacting with fluid. In order to handle rigid body dynamics in Immersed boundary method suitable modifications such as discrete forcing approach were proposed [3]. Both, the Level-Set and Volume-of-Fluids methods are interface tracking algorithms. An advection equation is solved for a signed distance function in level set method and fraction function is employed in case of the volume of fluids [4] [5]. Perhaps the simplest and most versatile approach to allow structure motion inside a fluid domain is overset mesh or Chimera grid technique which was first introduced in 1980's. In this approach, fluid domain and the surrounding structure are meshed independently. The overset method allows solid mesh to overlap with fluid mesh and the method keeps track of which mesh to use in which part of the domain [6]. Solid grid glide along the structural grid without any mesh deformation. This implies that mesh quality is always preserved using this technique. The only downside is possible increase in computation cost because of a communication and interpolation between solid and fluid mesh. Also, there may be some loss in accuracy due to interpolation error. Despite these limitations, the structural motion freedom allowed by overset methods totally outweighs these drawback, which can be overcome by using slightly more computational time, or finer grids, or higher order interpolation algorithms [7] [8].

Despite its potential superiority to handle moving boundary problems, overset grids did not took off as well as it should have among the CFD users. This is almost never taught in a graduate level CFD course and not a single textbook is available to train new generation CFD code developers. This slow adaptation of overset mesh by CFD community is perhaps due to its overwhelming complexity. Until recently, only few academic in-house codes and fewer commercial CFD software's have reported successful development of overset mesh [9] [10] [11]. A very recent development in CFD community is the release of overset mesh support in the new version of OpenFOAM, a popular open source Finite Volume discretization code for solving Partial Differential Equations (PDEs). This report is a validation study of this newly introduced OpenFOAM overset function. Flow around NACA0018 airfoil is simulated and the predicted lift, drag, moment coefficients are compared with experimental data. It is part of an ongoing project to simulate force induced rotation of vertical axis wind turbines. A validation study with a benchmark problem is necessary to test the accuracy and stability of the code.

\section{Problem geometry and Governing equations}

The rectangle in Fig. 1 represents a wind tunnel test section with the left face acting as an inlet and the right face being an outlet. The top and the bottom face of the rectangle represent sides of virtual wind tunnel. The obstacle in the middle is a cross section of NACA0018 airfoil. The objective is to simulate flow past the airfoil and to calculate the aerodynamic lift, drag and the moment coefficients. This is a two dimensional set up, which is equivalent to saying the setup in Fig. 1 is extruded infinitely along the positive $z$ as well as the negative $z$ direction. The exact dimensions of this geometry are listed in Table 1. This fluid flow domain 
dimensions are similar the dimensions used in the experimental setup by W.A. Timmer [12]. The airfoil is placed closer to the inlet at a distance of $0.8 \mathrm{~m}$ in order to visualize the flow separation in the near and far wake region.

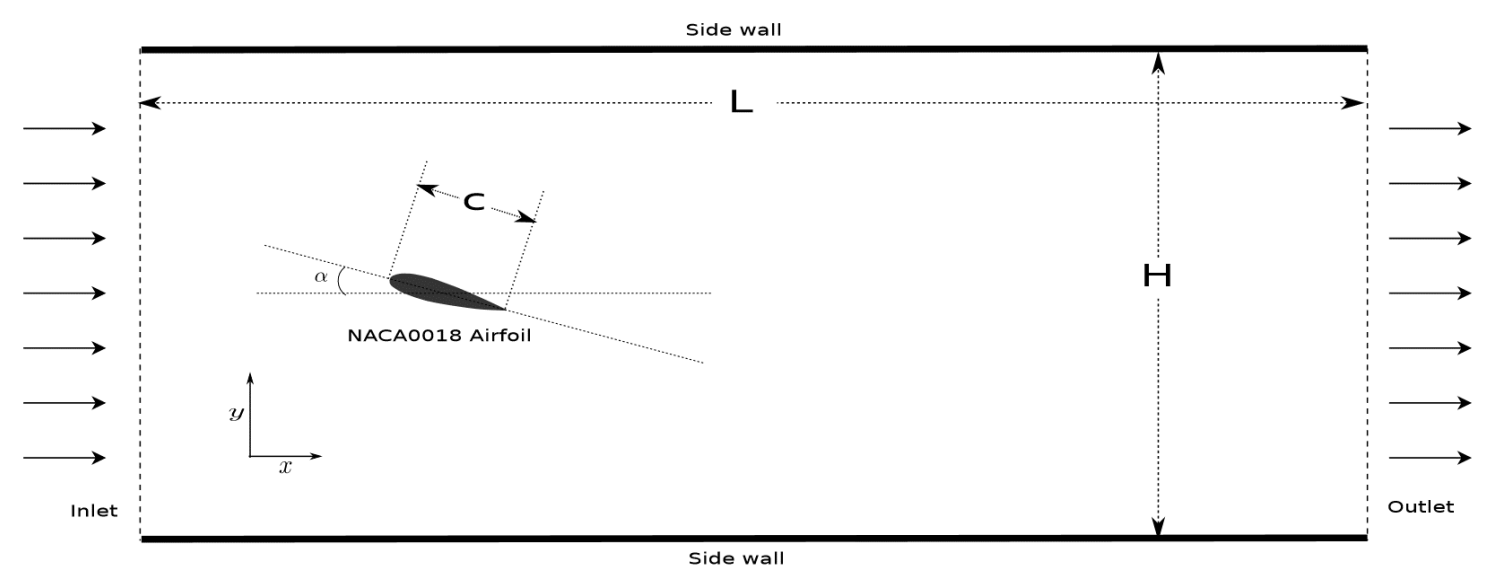

Figure 1. Schematic diagram of the domain and boundary

The lift, drag and moment forces were calculated by solving a steady-state Reynolds Averaged Navier-Stokes (RANS) equations modelled with $k-\varepsilon$ turbulence model. The $k-\varepsilon$ turbulence model is the most widely used model for industrial studies [13] with good convergence rate. This model performs well for flow around complex geometries and external flows with low memory requirements $[14,15]$.

Table 1. Specification for the test case

\begin{tabular}{c|c}
\hline Dimension & Value \\
\hline Channel Height, H & $1.25 \mathrm{~m}$ \\
Channel Length, L & $2.6 \mathrm{~m}$ \\
Airfoil Profile & NACA 0018 \\
Chord Length, c & $0.25 \mathrm{~m}$ \\
Reynold Number, Re & $3 \times 10^{5}, 5 \times 10^{5}$ and $7 \times 10^{5}$ \\
Angle of Attack & $0^{\circ}, 5^{\circ}, 10^{\circ}, 15^{\circ}, 20^{\circ}, 25^{\circ}$ and $30^{\circ}$ \\
\hline
\end{tabular}

$\nabla \cdot \mathrm{u}=0$

$\nabla \cdot(\mathrm{uu})=-\nabla p+\left(v+v_{t}\right) \Delta \mathrm{u}$

$\nabla \cdot(\mathrm{u} k)=\nabla \cdot(\nu \nabla k)=v_{t}\left[\frac{1}{2}\left(\nabla \mathrm{u}+\nabla \mathrm{u}^{T}\right)\right]^{2}-\varepsilon$

$\nabla \cdot(\mathrm{u} \varepsilon)=\nabla \cdot(\nu \nabla \varepsilon)=C_{1} \frac{\varepsilon}{k} v_{t}\left[\frac{1}{2}\left(\nabla \mathrm{u}+\nabla \mathrm{u}^{T}\right)\right]^{2}-C_{2} \frac{\varepsilon^{2}}{k}$

Eq. (1) governs the flow of mass (Eq. 1a), momentum (Eq. 1b), turbulence kinetic energy, k (Eq. 1c), and turbulence dissipation rate, $\varepsilon$ (Eq. 1d) defines the rate at which energy is dissipated [16]. The variable $\mathbf{u}$ is the fluid velocity field, $p$ is the effective pressure, that means $p=$ 
$\frac{\text { pressure }}{\text { density }}+\frac{2}{3} k$ and $v$ is the kinematic viscosity of the fluid. The coupling between the variables $k, \varepsilon$ with $\mathbf{u}$ and $p$ is given by the turbulent kinematic eddy viscosity is computed as

$$
v_{t}=C_{\mu} \frac{k^{2}}{\varepsilon}
$$

And the model constants $C_{1}=1.44, C_{2}=1.92$ and $C_{\mu}=0.09$. The lift $(L), \operatorname{drag}(D)$ and moment $\left(M_{z}\right)$ on the airfoil are calculated using (Eq. 3), (Eq. 4), and (Eq. 5) respectively [12].

$$
\begin{aligned}
L & =\oint_{S}(\sigma \cdot \hat{n}) \cdot \hat{\mathrm{e}}_{y} d s \\
D & =\oint_{S}(\sigma \cdot \hat{n}) \cdot \hat{\mathrm{e}}_{x} d s \\
M_{z} & =\oint_{S}\left[(\sigma \cdot \hat{n}) \times\left(\hat{x}-\hat{x}_{c}\right)\right] \cdot \hat{\mathrm{e}}_{z} d s
\end{aligned}
$$

$\sigma$, the stress tensor is expressed as

$$
\sigma=p \mathrm{II}+\mu\left(\nabla \mathrm{u}+\nabla \mathrm{u}^{T}\right)
$$

where $\widehat{\boldsymbol{x}}_{c}$ is the aerodynamic center, $\nabla \mathrm{u}$ denotes the velocity gradient and $\mu$ is the viscosity coefficient. The non-dimensional aerodynamic force coefficients on the airfoil are calculated according to the formulae given below [18]:

$$
\begin{gathered}
\text { Lateral lift coefficient } C_{L}=\frac{L}{0.5 \rho A U_{\infty}^{2}} \\
\text { Longitudinal drag coefficient } C_{D}=\frac{D}{0.5 \rho A U_{\infty}^{2}} \\
\text { Moment coefficient } C_{M}=\frac{M}{0.5 \rho A l U_{\infty}^{2}}
\end{gathered}
$$

Here $\rho$ defines the density of the working fluid, $A$ reference area of the airfoil and $U_{\infty}$ freestream air velocity. Standard atmospheric air properties are selected for the study with an air density $\rho=1.225 \mathrm{~kg} / \mathrm{m}^{3}$ and $v=1.48 \times 10^{-6} \mathrm{~Pa} / \mathrm{s}$. The Reynolds number Re for flow past airfoil is calculated using Eq. (10)

where $l$ is the chord length of the airfoil.

$$
R e=\frac{U_{\infty} l}{v}
$$

Boundary conditions used in this study are explained next for each boundary segment.

- Inlet: Uniform inlet velocity $\left(U_{\infty}\right)$ is set with respect to the calculated Reynolds number Re, while the pressure is treated with Neumann boundary condition $\left(\frac{\partial p}{\partial n}\right)=0$. For 
turbulent kinetic energy $(k)$ and turbulence dissipation rate $(\varepsilon)$, a fixed value constraint is supplied which is the calculated energy in turbulence and its dissipation with respect to the inlet velocity given by $k=\frac{3}{2}\left(U_{\infty} I\right)^{2}$ and $\varepsilon=\frac{0.164 k^{1.5}}{0.007 L}$, where Turbulence intensity $I=0.16(R e)^{-0.125}$ and Turbulence length scale $L=C_{\mu} \frac{k^{\frac{3}{2}}}{\varepsilon}[19]$.

- Outlet: For the outlet, $p$ is set to Dirichlet condition $p=0$. All other variables $\mathbf{u}, k$ taken Neumann boundary condition at the outlet.

- Airfoil: A no-slip $(\mathbf{u}=0)$ condition for velocity field; The variables $p, k$ and $\varepsilon$ are treated with zeroGradient condition [20][21].

- Top and bottom: Velocity field is treated with Dirichlet condition $\mathbf{u}=0$. Other variables have the same boundary conditions as in the airfoil boundary segment.

\section{Overset Mesh Details}

This section details the mesh used in this study. Our overset mesh is basically two curvilinear structured mesh overlapping with each other (Fig. 2). One set of mesh is created around the airfoil by partitioning the airfoil curve into line segments, then forming layers in the outward normal direction (Fig. 2 top right). We will refer to this curvilinear mesh around the airfoil to as the front-mesh. The boundary layer flow around the airfoil will be resolved using this mesh. Another set of mesh cells are generated by a straightforward rectangular discretization of the background rectangular domain. The whole mesh generation process is automatized. A python script reads NACA0018 coordinates and produces a file in a format accepted by a mesh generation software Gmsh 3.0.1 [22]. Gmsh then produces the overset mesh as seen in Fig. 2 (the bottom subfigure). Around 1.5 million hexahedral cells have been generated out of which 22680 are overset cells around the airfoil surface and 1444203 elements are for the fluid domain.

In order to capture the appropriate boundary layer near the airfoil wall and the influence of Reynolds stress tensor the first cell center near the airfoil needs to be sufficiently close. According to procedure suggested in [23], the first cell center near the airfoil is kept at a distance of $y_{\perp}=0.4$. For our chosen turbulence intensity $I=0.02$, this value of $y_{\perp}$ corresponds to maintaining the non-dimensional wall distance $\mathrm{y}^{+}=11$, where $y^{+}=\frac{U_{f} \times y_{\perp}}{v}$, where $U_{f}=\sqrt{\frac{\tau_{w}}{\rho}}$ is the friction velocity. The variable $\tau_{w}=0.5 U_{\infty}^{2} \rho C_{f}$ is the wall shear stress with $C_{f}=$ $0.0576 \operatorname{Re}^{-0.2}$ defines the local skin friction coefficient [20]. 

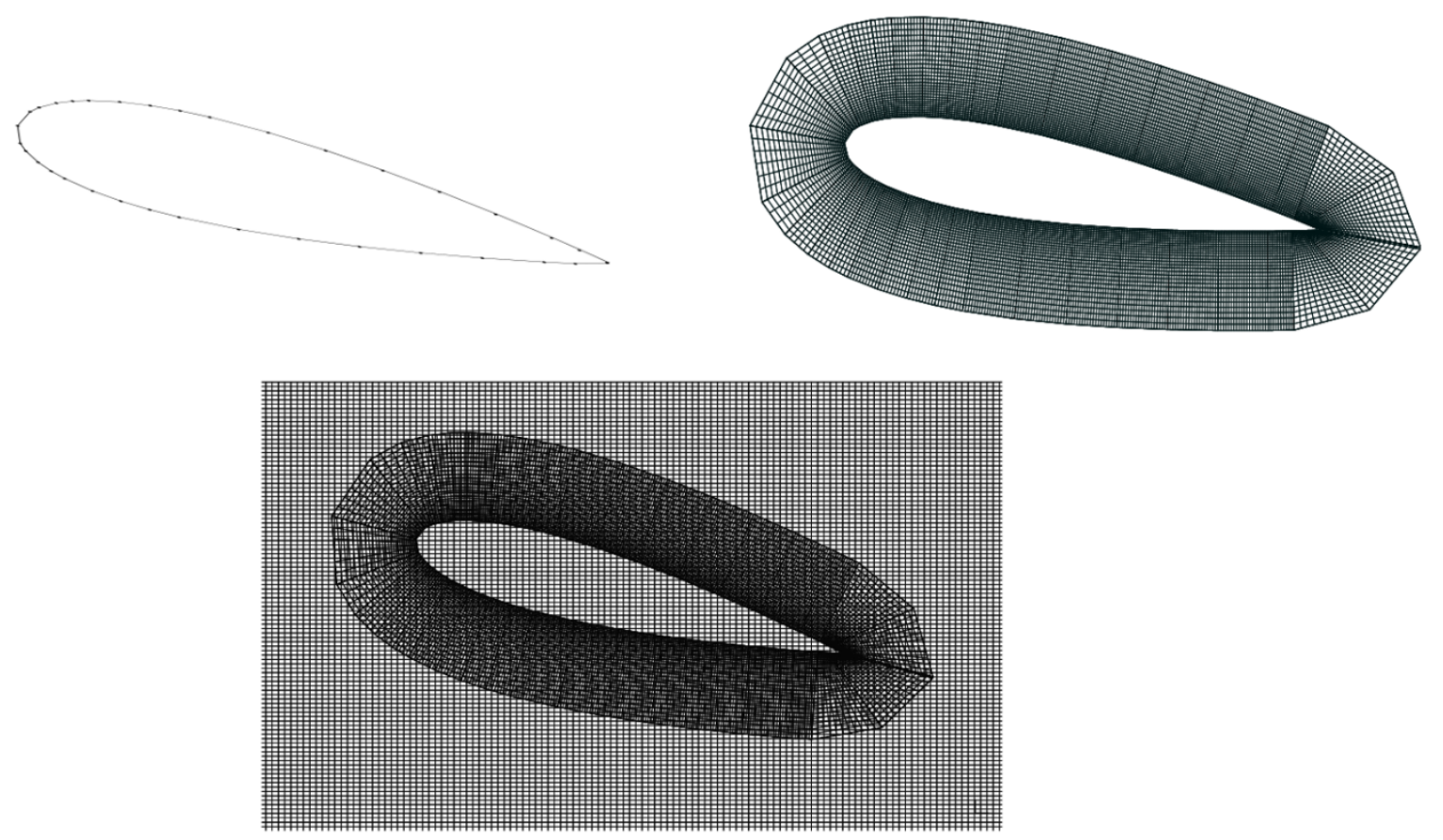

Figure 2. Layout of airfoil grid overlapping with the domain grid.

\section{Finite volume discretization of Governing equations with overset mesh}

The overset mesh idea is basically applying FVM discretization with two sets of overlapping cells. Near the airfoil, front mesh is used and, away from the airfoil and near the tunnel boundary, the background mesh is used. In the region of intersection between two meshes, values from one mesh gets interpolated to the other. The below subsection briefly explains about this process.

Hole Identification: The first task in solving a PDE with an overset mesh is to remove a set of cells which are not going to be used in the calculation. These are called the hole cells. In the present case, the intent is to use the front mesh (the cell layers around the airfoil) for resolving flow near airfoil, and since the airfoil is in-penetrable, any cell inside airfoil is irrelevant. The holes here will be a set of cells from the background mesh which are either inside the airfoil or are near to the airfoil. After removal, we are left with non-hole cells as shown in Fig. 3.

Acceptor Cells: After removal of hole cells, the next step is to identify a set of acceptor cells (the red colored cells in Fig. 4). These cells mark the boundary of one cell zone overlapping with other cell zone. The red cells in Fig. 4 (top-left) denotes the boundary for background mesh. Similarly the red cells in Fig. 4 (bottom-left) are the acceptors cells from the front mesh zone, representing the boundary of front mesh in the overlapping region. Equations are not discretized at acceptor cells, rather they act as ghost cells for finite volume discretization of the cells adjacent to them [24]. 

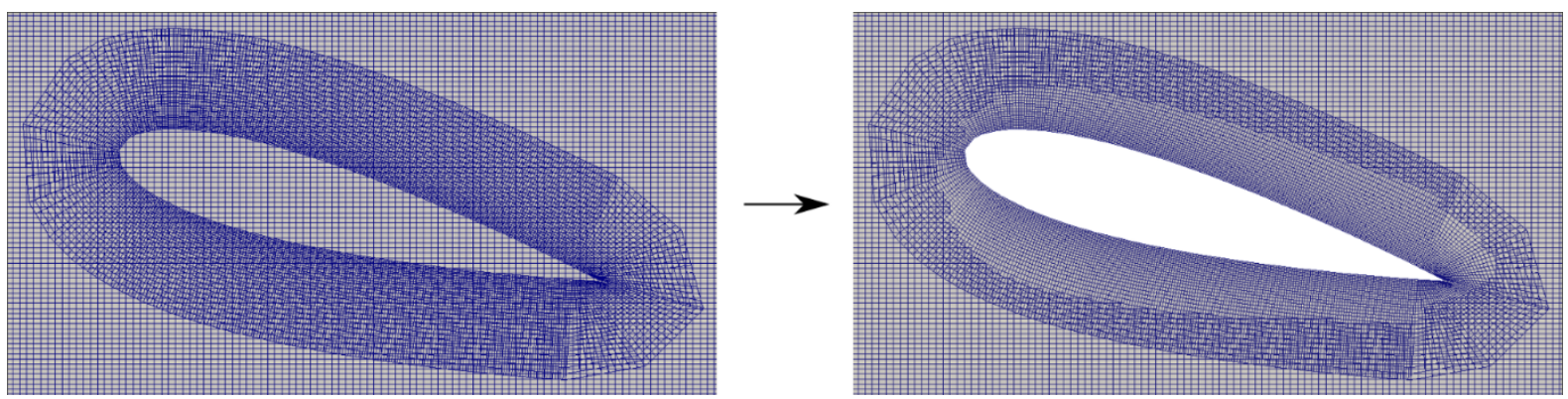

Figure 3. Starting mesh to actual working mesh obtained by removing unwanted cells from background mesh cells which were either lying inside the airfoil or were too close to the airfoil.

Donor Cells: Though the equations are not discretized at acceptor cells, still the variables at acceptors cells center enter into discretized equations during FV discretization of equations in the cells adjacent to the acceptor cells. This leads to a theoretical gap between the number of equations and the number of variables. And we are short of exactly same number of equations as there are number of acceptor cells. This gap in the number of equations is completed by interpolation equations for values of flow variables at acceptor cell centers in terms of flow variables of the overlapping cells. To save computational cost, OpenFOAM pre-defines a set of cells which it calls donor cells and these are the only cells which get used in creating the interpolating equations. The blue cells in Fig. 4 shows the pattern of donor cells from overlapping cells used in this study.
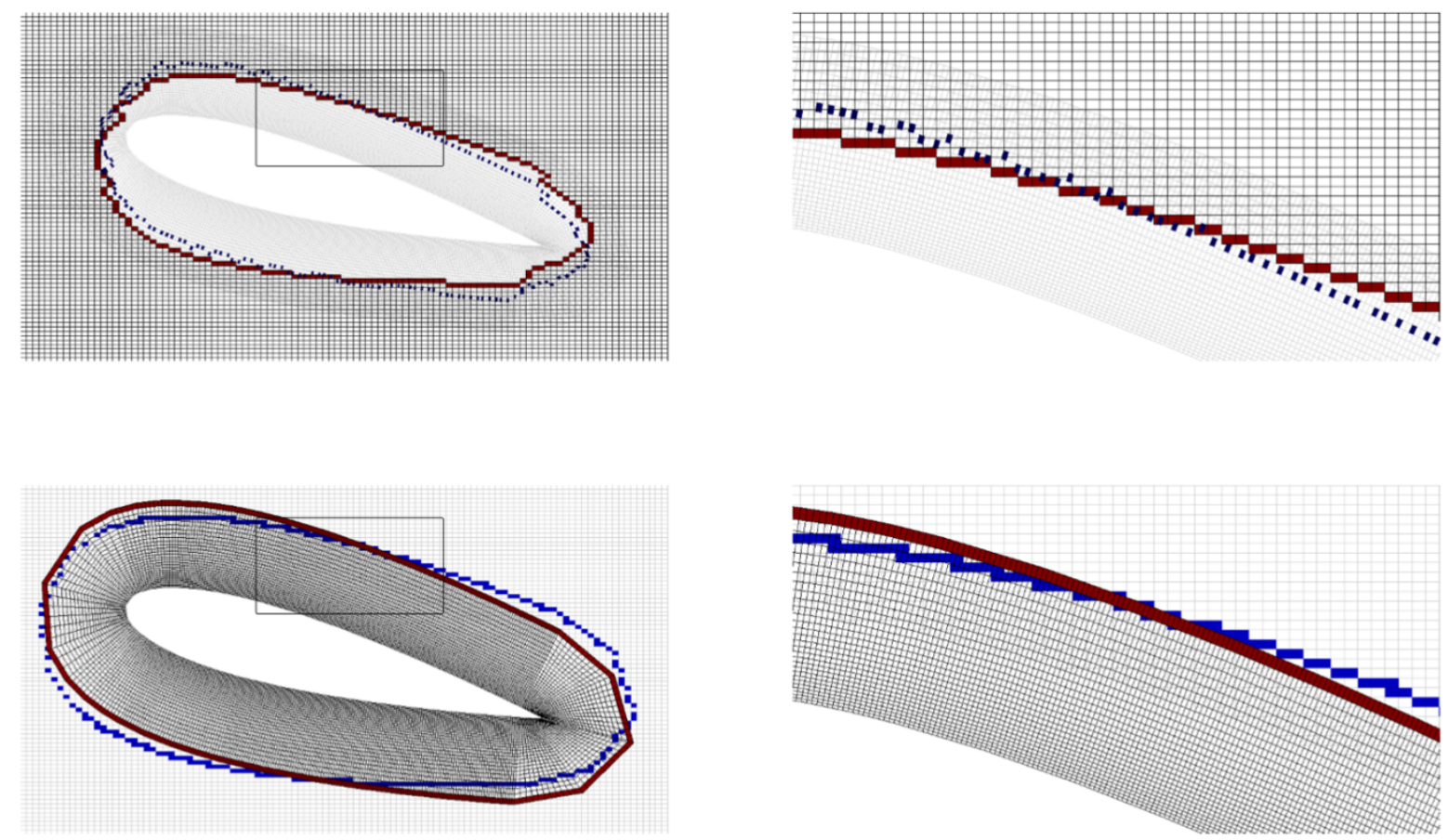

Figure 4. Acceptors and donor cells. The top-left figure is showing the choice of acceptors (red) from the background mesh and the donor (blue) from the front mesh. The top-right figure is a zoomed-in image of top-left. The bottom-left figure is showing the choice of acceptors (red) from the front mesh and the donor (blue) from the background mesh. The topright figure is a zoomed-in image of top-left. 


\section{OpenFOAM details and Solver settings}

This section gives details about different solver and settings of OpenFOAM we have used in this project. The version foam-extend-4.0 is used for this study. This is a development version of the CFD library and does not automatically come with overset mesh support. The overset mesh functionality is available separately, which also is open-source and is free. The Navier Stokes equations is solved using the module overSimpleFoam, a solver for steady state flow with overset mesh support. The overSimpleFoam discretizes the steady-state incompressible Navier-Stokes equation, coupled with $k-\varepsilon$ turbulence model, using the SIMPLE algorithm [25, 26]. Various schemes used to discretize each term are listed in the Table 2. The resulting linear equations are solved using PBiCGStab (Preconditioned Stabilized Biconjugate) solver with Diagonal Incomplete LU (DILU) preconditioner for elliptical equations and smooth solver with symGaussSeidel for the transport equation [27]. Flow fields are updated in under-relaxed fashion and following factors are used: pressure 0.3 , velocity 0.7 , epsilon 0.5 , and $k 0.5$. The residual levels for the iterative process are set to $10^{-6}$ as a convergence criterion for the pressure, velocity and turbulence field [28]. Fig. 5 shows decaying plot of residuals with respect to time. The graph clearly shows that solutions are converging well with our chosen solution parameters.

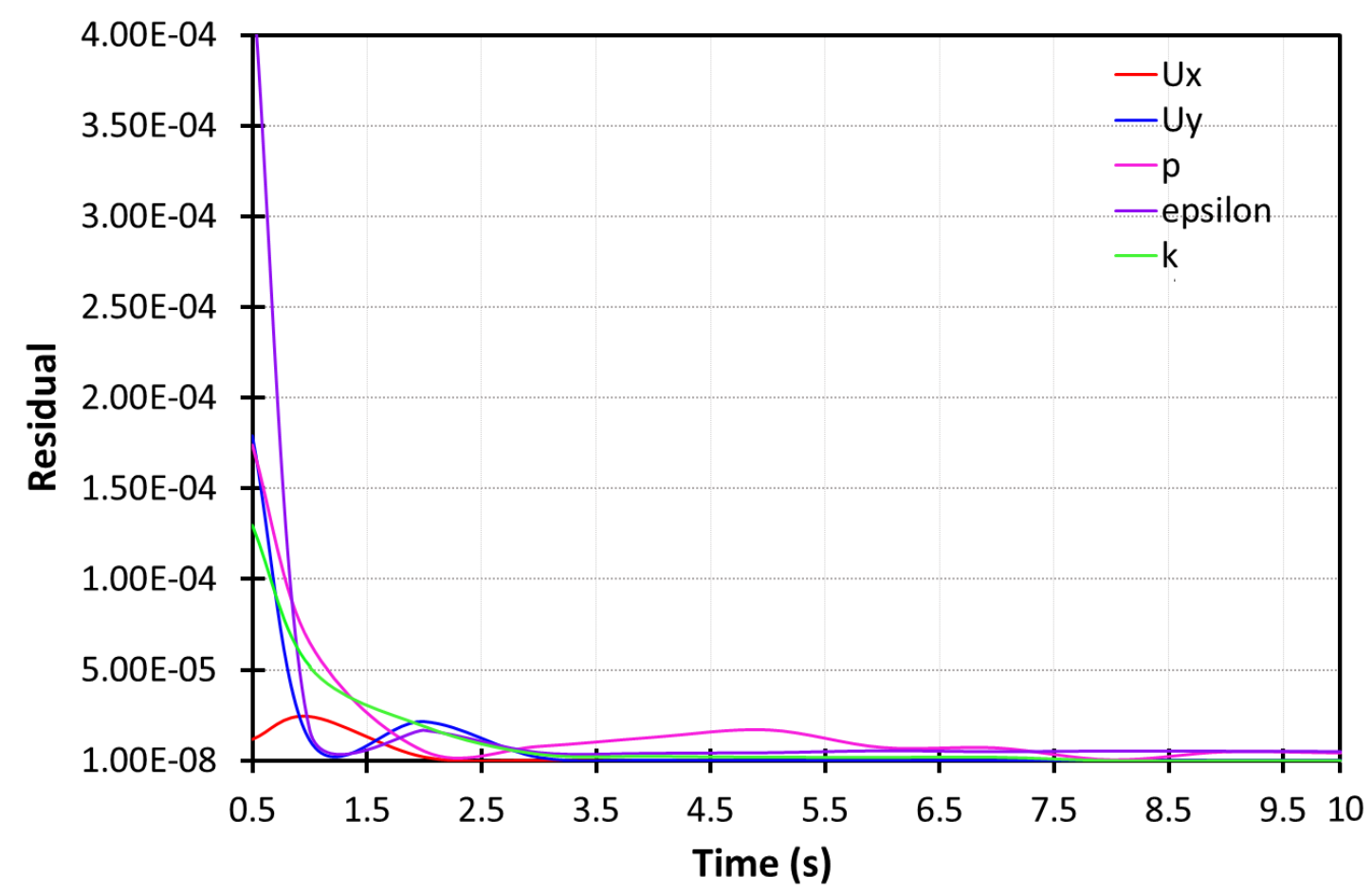

Figure 5. Time history of residuals at $\operatorname{Re}=3 \times 10^{5}$

Table 2. List of Numerical schemes selected for the present study.

\begin{tabular}{c|c}
\hline Terms & Discretization Scheme \\
\hline Time scheme $\frac{\boldsymbol{\partial}}{\boldsymbol{\partial} \boldsymbol{t}}$ & Steady-state (time derivatives to zero) \\
Divergence $(\boldsymbol{\nabla})$. & Second order, Upwind-biased \\
Gradient $(\boldsymbol{\nabla})$ & Second order, Gaussian integration \\
Laplacian $\left(\boldsymbol{\nabla}^{\mathbf{2}}\right)$ & Gaussian integration \\
Interpolation & Linear interpolation \\
\hline
\end{tabular}




\section{Results and Discussion}

The main objective of this study is to simulate a flow past NACA 0018 airfoil using OpenFOAM's new overset mesh feature and validate with a benchmark experimental data available in [12]. A parametric study using overset mesh functionality is performed for three different Reynolds number $\operatorname{Re}\left(3 \times 10^{5}, 5 \times 10^{5}\right.$ and $\left.7 \times 10^{5}\right)$ and seven different angle of attack $\alpha$ $\left(0^{\circ}-30^{\circ}\right)$ with an increment of $5^{\circ}$. In the following section, the coefficient of pressure predicted on the upper surface and lower surface of the NACA 0018 airfoil is presented and analyzed in detail. And later on, the calculated aerodynamic coefficients are plotted against experiments and comparison between them is analyzed.

\subsection{Comparison between calculated and experimental Pressure Coefficients}

The pressure distribution along the surface of an airfoil is defined by a dimensionless number: pressure coefficient. The pressure coefficient, for a two-dimensional airfoil with a fixed angle of attack is given by the formula

$$
C_{p}=\frac{p-p_{\infty}}{0.5 \rho U_{\infty}}
$$

where $p$ is the static pressure and $p_{\infty}$ is the freestream pressure or reference pressure [30]. The pressure coefficient is defined at each point of the airfoil. The difference in pressure in the upper and lower surface of the airfoil generates upward lift force [31]. As the angle of attack increases, these pressure variations also tend to increase with a significant high suction peak at the leading edge which keep reducing at the trailing edge of the airfoil. The conventional way of plotting the coefficient of pressure distribution is followed in Fig. 6, where the negative peak value represents the pressure on the upper surface of the airfoil suction side and positive peak on the lower surface. The difference in the two peaks is higher on the leading edge which is the main contributor for lift, and this variation keep reducing from the mid chord till the trailing edge. The dimensionless coefficient of pressure $C_{p}$ calculation is performed with an angle of attack, $\alpha=8^{\circ}$ and Reynolds number, $R e=7 \times 10^{5}$. Fig. 6 shows the difference in pressure coefficient $C_{p}$ which suggests that as the angle of attack is increased, the pressure coefficient on the upper surface of the airfoil remains negative while on the lower surface remains positive. The overset grids clearly captures the flow separation on the airfoil suction side. The gradual decrease in $C_{p}$ value on the suction side as well as the positive value of $C_{p}$ on the pressure surface are also well predicted. Also, the increase in angle of attack predicts a little adverse in pressure gradient in the suction side. From Fig. 6 peak pressure is predicted at 0.02 of chord at the leading edge of the airfoil denoting the main contributor for lift while at the trailing edge it falls drastically. The peak pressure coefficient is measured from around $0 \%$ to $5 \%$ of the chord length by the overset mesh. In addition, the pressure recovery phase is clearly captured in the suction side downstream which changes with the flow condition near the trailing edge due to separation bubbles and difference in pressure gradient. Overall the pressure difference between the suction side and the pressure side of the airfoil is well predicted and this variation is quite minimal providing increase in lift at this angle of attack. 


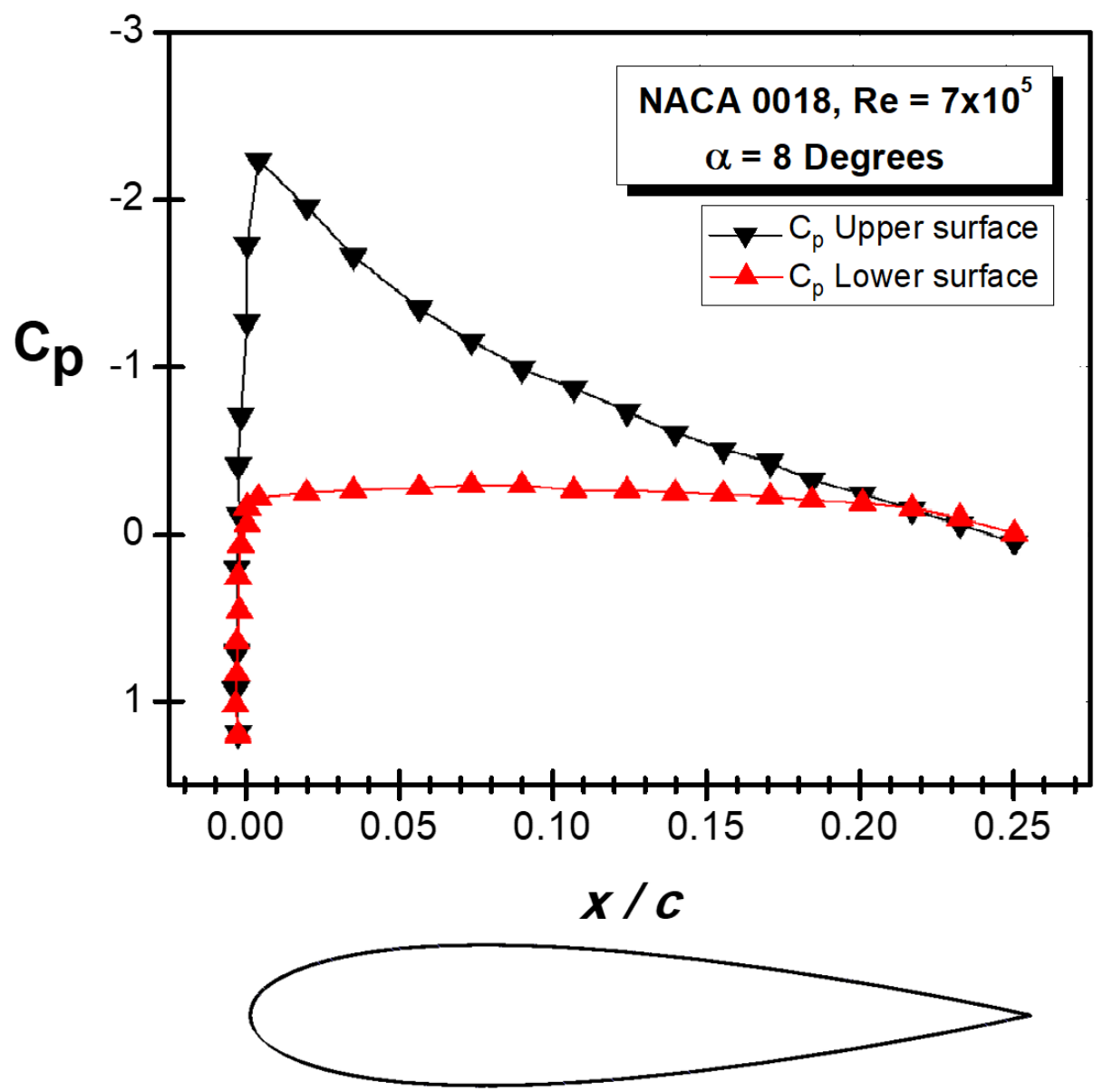

Figure 6. Coefficient of pressure on the surface airfoil. Positive pressure coefficient on the lower surface indicates that the pressure on the pressure-side of the airfoil exceeds atmospheric pressure. The negative pressure coefficient on the lower surface indicates that the pressure on the suction side is in proportion to the atmospheric pressure.

\subsection{Distribution of the aerodynamic coefficient at $R e=300000$}

Fig. 7 shows the lift, drag and moment coefficient comparison on the airfoil surface with experimental study at $\mathrm{Re}=3 \times 10^{5}$ for seven different angles of attack, $\alpha$. The overset mesh and $k-\varepsilon$ turbulence model adequately predicts the lift, drag and moment coefficient plots which shows a reasonable trend with that of the experimental wind tunnel test conducted by W.A. Timmer [12]. Until $\alpha=15^{\circ}$, the lift curve is linearly increasing and drag remains low because the flow was more attached towards the wind direction. The lift curve shows a steady plateau phase as the airfoil angle of incidence increases. Beyond $\alpha=15^{\circ}$, there is a constant decline in the lift and a steady increase in drag. This regime is known as the stall zone. The separation bubble forms at the trailing edge and start to move forward to reduce the lift $[32,33]$. The results also suggest that the overset mesh is able to predict the stall regime where the flow separation occurs and gradually moves towards the airfoil upstream. In the post stall regime, flow separation takes place with two-bubble separation that can be visibly seen in the contour plot in Fig. 13. Single large bubble forms downstream towards the leading edge and a small bubble forms at the trailing edge. This results in huge wake vortices in the domain, leading to the increase in drag coefficient [34]. The drag coefficient remains minimal till $\alpha=10^{\circ}$, later there is a sudden surge in drag coefficient. Beyond stall angle $\alpha=20^{\circ}$, the lift curve tend to increase again and reaches a maximum value as lift curve follows the sine curve expect the 
stall regime. The coefficient of drag is always directly proportional to angle of attack. The drag curve increases monotonously with the increase in angle of attack $\alpha$. Simulation results suggests that the pitching moment coefficient for the given airfoil remains zero between $\alpha=0^{\circ}$ to $\alpha=5^{\circ}$, while at higher angle of attack, it falls to negative. The plot also clearly shows that there is very little variation in the airfoil moment coefficient with respect to increasing angle of attack as the value of pitching moment of a symmetrical airfoil always stays very close to zero. The decaying slope in the plot indicates the positive static stability of the airfoil. In summary, all the three plots shows a reasonable trend and agreement with the experimental results in terms all the predicted aerodynamic coefficients. Small discrepancies in the plot are due to blockage effect that occurs in wind tunnel testing or may be the experimental flow field may not be fully turbulent when compared to fully turbulent. Also the absence of three dimensional effect on the airfoil span-wise flow and tip vortices [35].
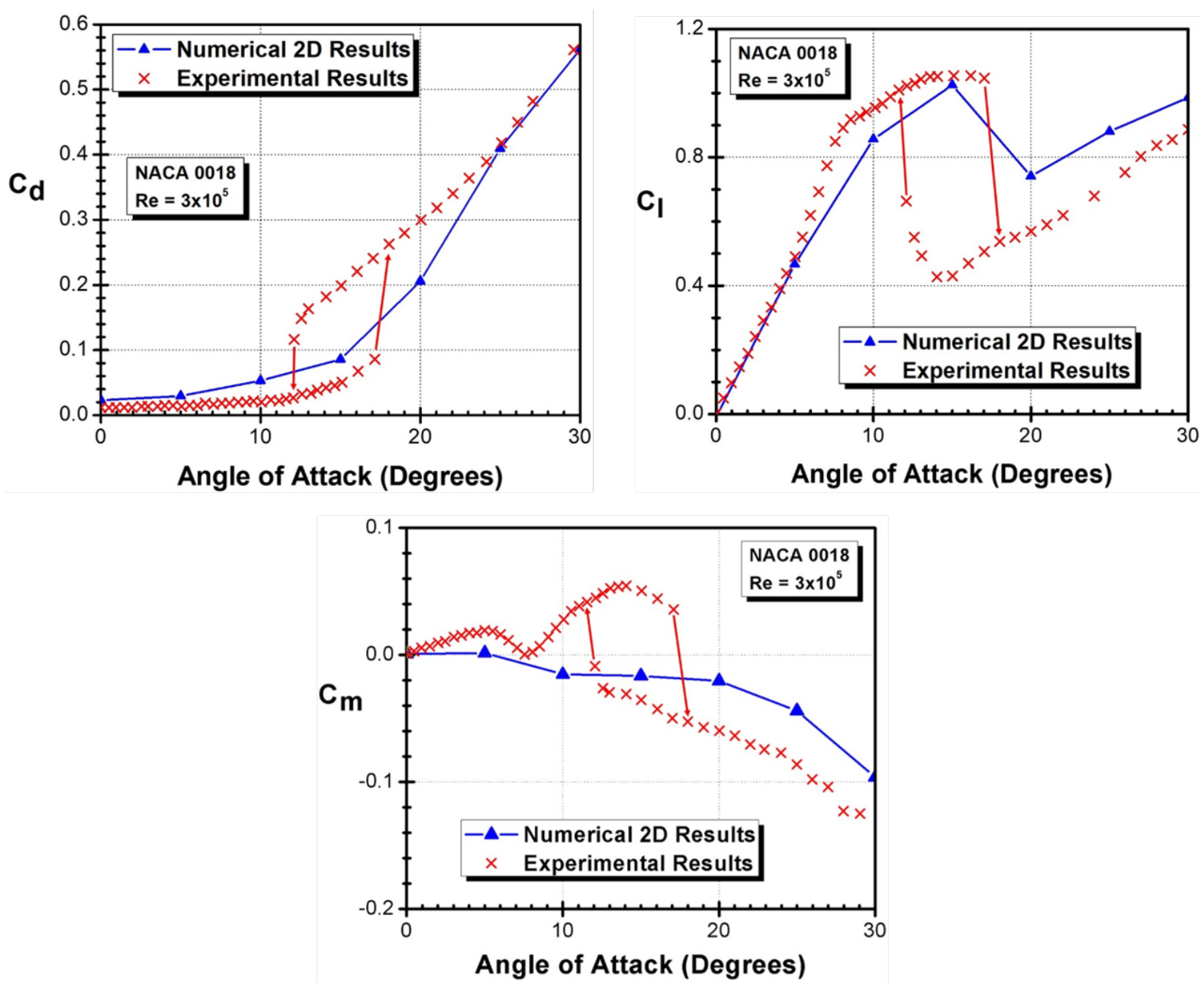

Figure 7. Effect of Reynolds number on (a) drag (b) lift and (c) moment at $\operatorname{Re}=3 \times 10^{5}$ 


\subsection{Distribution of the aerodynamic coefficient at $R e=500000$}

The results for $\mathrm{Re}=5 \times 10^{5}$ show similar trend with that of $\mathrm{Re}=3 \times 10^{5}$. The coefficient of lift and drag increases with increase in Reynolds number until the stall regime. In Fig. 9, we observe that the lift plot correlates with the experimental data with a smooth spline curve until $\alpha=10^{\circ}$. After $\alpha=15^{\circ}$, the curve predicts the stall regime. Separated bubbles from the leading and trailing edge of the airfoil tend to decrease the lift coefficient significantly. In addition, as the Reynolds number is increased, the intensity of the separated bubble is also on the higher side and the flow transmits to the turbulence phase. After the stall regime, the coefficient of lift starts to increase due to the pressure recovery on the suction side. No significant change in the coefficient of drag plot which shows a gradually increase as the angle of attack increases. The moment, on the other hand, falls steadily from $\alpha=0^{\circ}$ and forms a slope until $\alpha=30^{\circ}$ which stabilizes the airfoil's aerodynamic center post stall. This phenomena is quite similar in symmetrical airfoil.

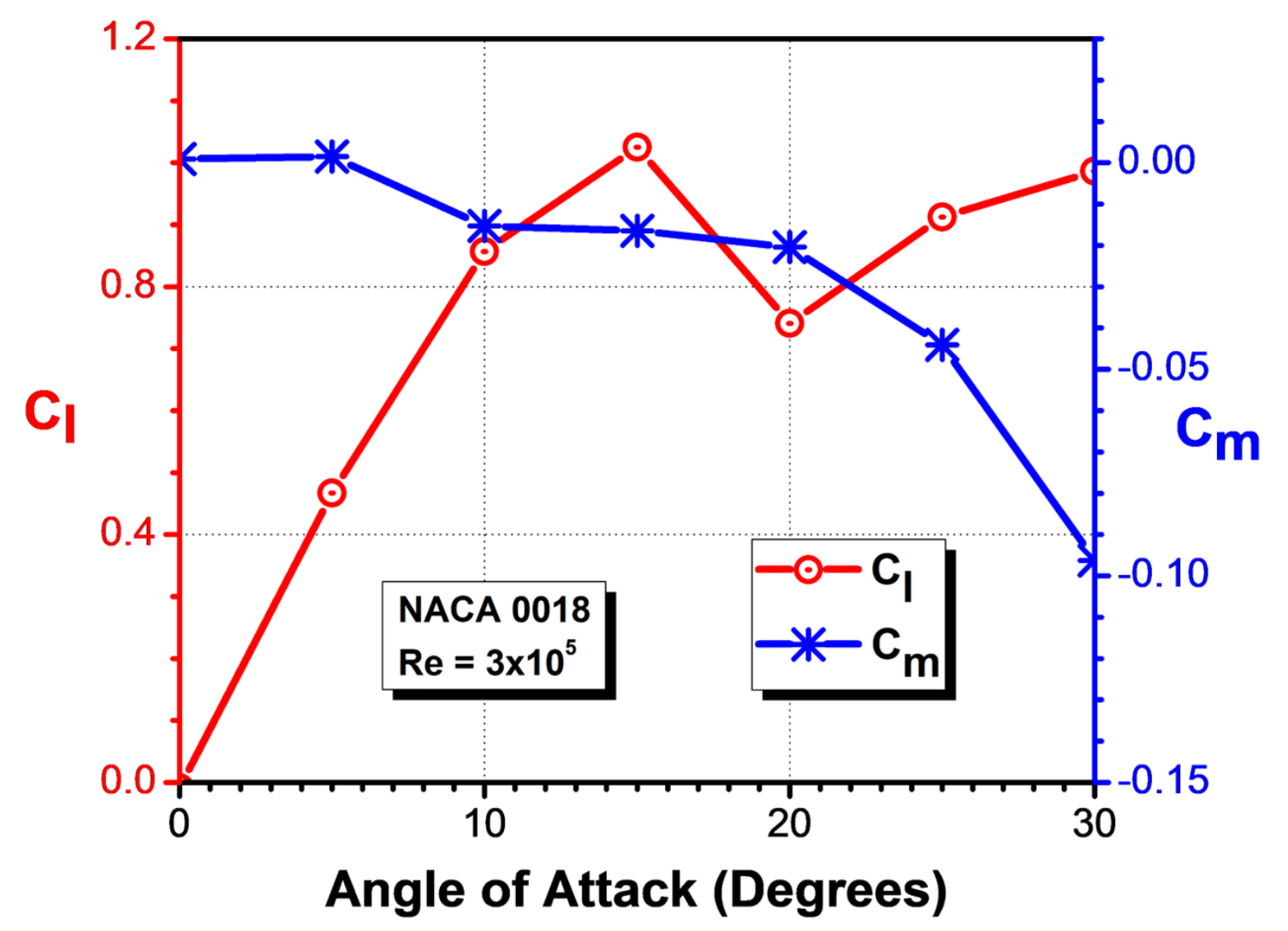

Figure 8. Effect of Reynolds number on lift and pitching moment coefficients at $\mathrm{Re}=3 \times 10^{5}$ 

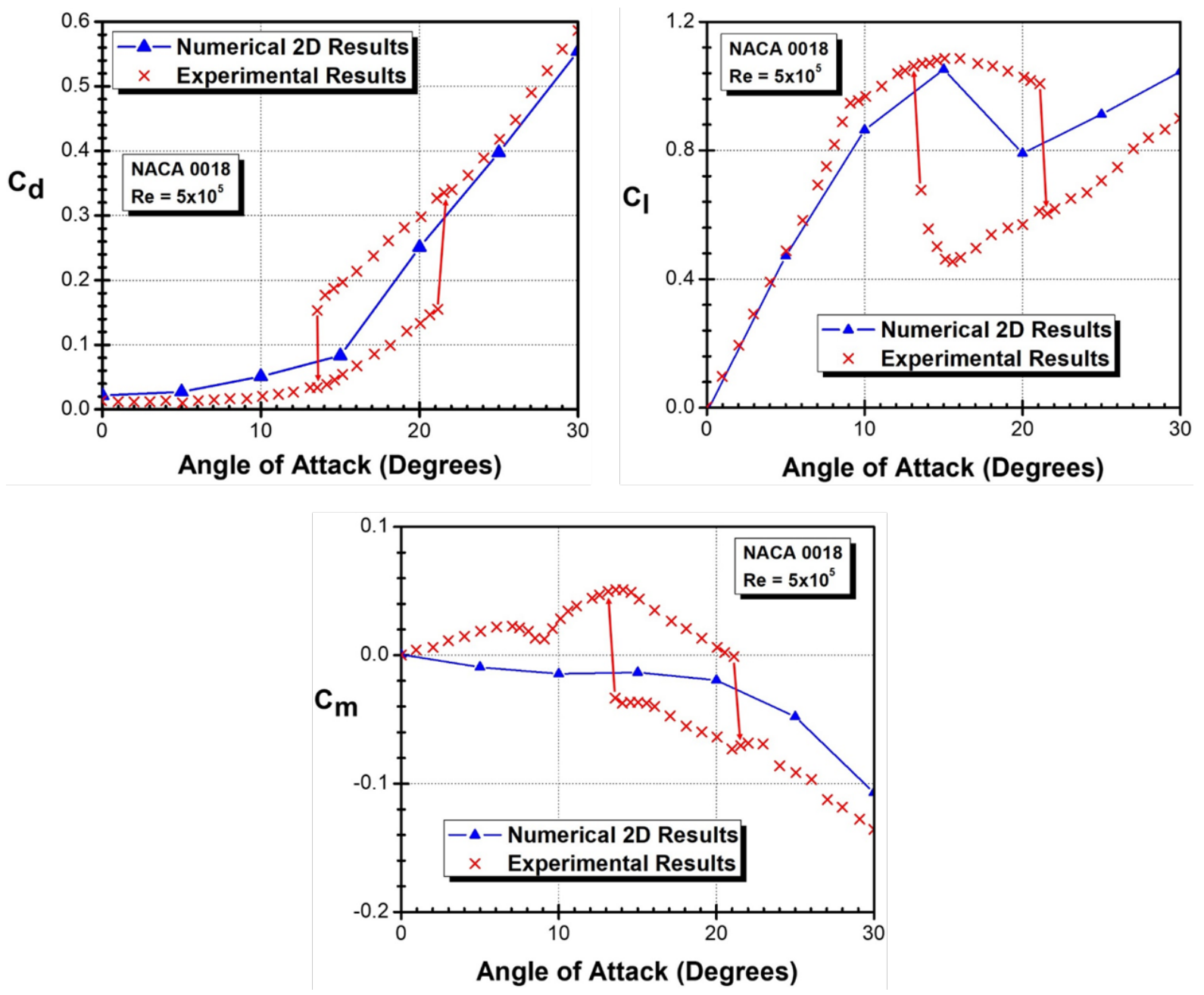

Figure 9. Effect of Reynolds number on (a) drag (b) lift and (c) moment at $\mathrm{Re}=5 \times 10^{5}$

\subsection{Distribution of the aerodynamic coefficient at $R e=700000$}

The lift and drag are also calculated for $7 \times 10^{5}$. In this case of increases in-flow velocity, the flow is completely attached to the upper and lower surface of the airfoil for low angle of attacks from $\alpha=0^{\circ}$ to $\alpha=15^{\circ}$ which can be observed from both the lift and drag plots. Further, stall delay is predicted as the flow velocity is increased proportional to the angle of attack. Increasing the angle of attack of the airfoil in a low Reynolds number flow results in aerodynamic stall [36]. The increase in pressure drag also pushes the transition phase from the suction side towards the leading edge developing a laminar separation after $\alpha=15^{\circ}$. 

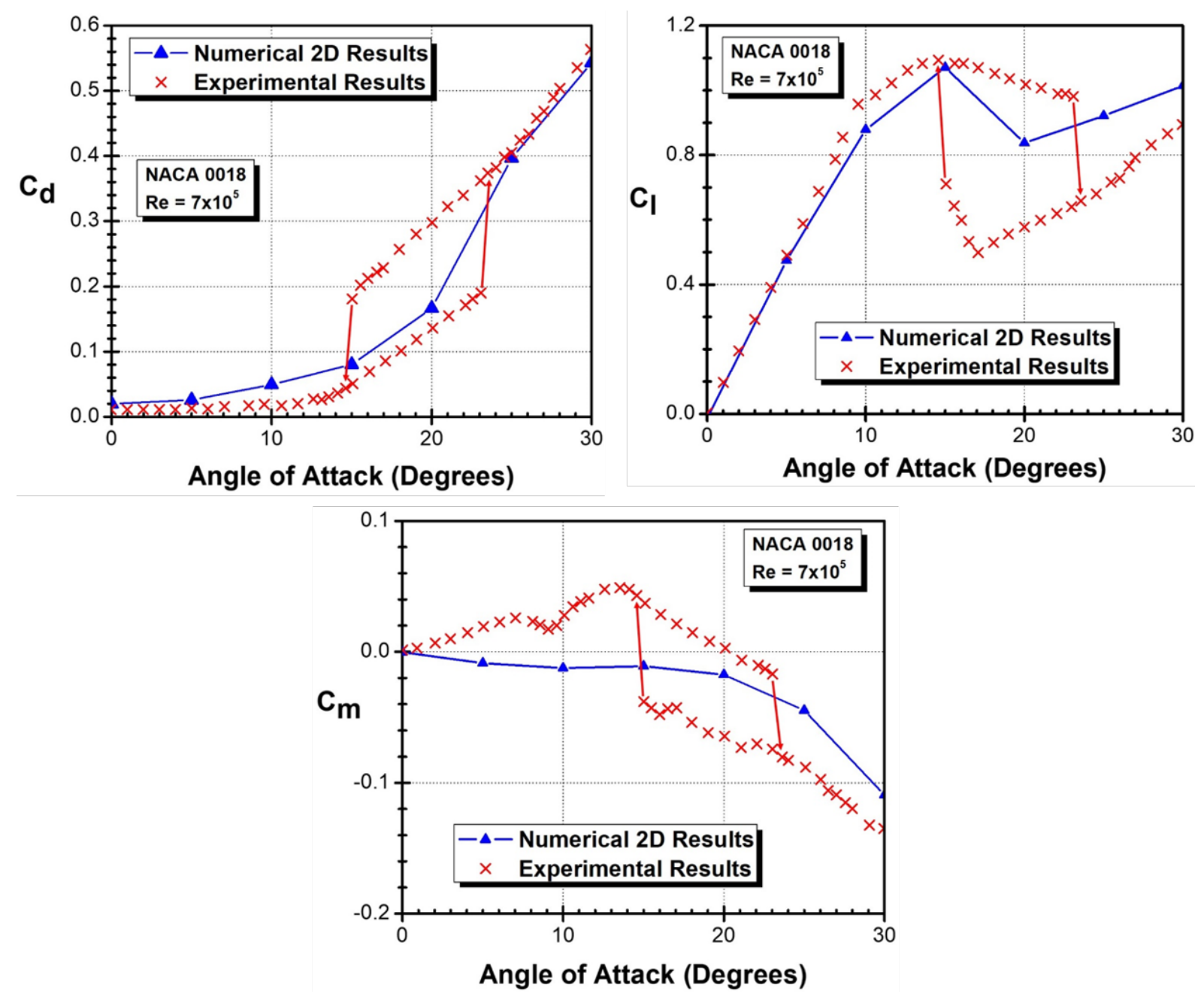

Figure 9. Effect of Reynolds number on (a) drag (b) lift and (c) moment at $\operatorname{Re}=7 \times 10^{5}$

Overall from the three different Reynolds number study, the overset grids shows the capability to model the complex flows over complex structures by predicting the exact trend in graph with the experimental results. From Fig. 11, we can observe that at $\alpha=0^{\circ}$, the flow over the airfoil is smooth, attached and symmetric creating a laminar slipstream on the surface that is visibly captured by the overset mesh. Later at $\alpha=5^{\circ}$, the flow is influenced by laminar separation bubbles building up turbulent regime in the suction region with strong adverse pressure gradient this region is called as the transition region. At $\alpha=10^{\circ}$, the flow is still attached to the airfoil surface with a thin laminar boundary layer separation. The thin boundary layer results in reduced flow velocity on the airfoil surface. When the angle of attack is increased to $\alpha=15^{\circ}$, the flow slowly detaches from the trailing edge, becomes asymmetric and gives rise to chaotic flow. The chaotic flows generates shallow separation bubble from the mid-section of the suction region that directly leads to the increase in drag on the airfoil which also results in predicting the stall with upward wake at the trailing edge. Again the flow velocity on the airfoil will be reduced due to friction. Generation of induced drag due to the vortices formed at the trailing edge of the airfoil at $\alpha=20^{\circ}$ reduces the lift further. Such vortices are called trailing vortices which are more visible. At $\alpha=20^{\circ}$, the flow gets completely detached from the airfoil leading edge and trailing edge. The overset mesh is able to capture the high-pressure curling of air pushing up from the leading and trailing edges of the airfoil leading to flow reversal over the airfoil surface. These counter rotating vortices at the trailing edge define the unstable boundary layer at the leading edge. When the angle of attack is further increased to $\alpha=25^{\circ}$ due 
to adverse pressure gradient the flow is reversed increasing the vortex strength at the leading and trailing edge. Later the vortices breaks into small vortices. At $\alpha=25^{\circ}$, one can visualize that these trailing edge vortices roll up towards the downstream wake region, a new trailing edge vortices forms. As discussed above in the $C_{L}$ and $C_{D}$ plots, up to $\alpha=10^{\circ}$, no evidence of the formation of separated bubbles in the flow is seen in the sequence of vorticity streamline plot as shown in Fig. 13. In fact, the flow is more attached and smooth until the dynamic stall regime is reached. In addition, one can clearly see that towards the downstream in the near wake zone no indication of wing tip vortices suggests the flow is in the laminar regime and there is no transition in flow. At about $\alpha=15^{\circ}$, the flow get detached from the trailing edge of the airfoil due to shear layer which in fact reduces the lift and develops separated boundary layer. In the stall regime the smooth laminar flow transforms to turbulent without the development of separation bubble that can be clearly seen in the streamline plot. However, beyond this angle the leading edge separation bubble develops [37]. At $\alpha=20^{\circ}$, the tip vortices from the leading edge become immense and travel along the whole length of the airfoil. The strength of this vortex will be huge with increased size and they stagnate near the suction surface of the airfoil hence reducing the pressure. The particular leading edge vortex will get detached from the airfoil and will be ready to shed when the airfoil experiences further increase in angle of attack [38]. Later on at $\alpha=25^{\circ}$, two separation bubbles burst out from both the leading edge and the trailing edge. They both collide each other and get separated away from the airfoil surface [39].
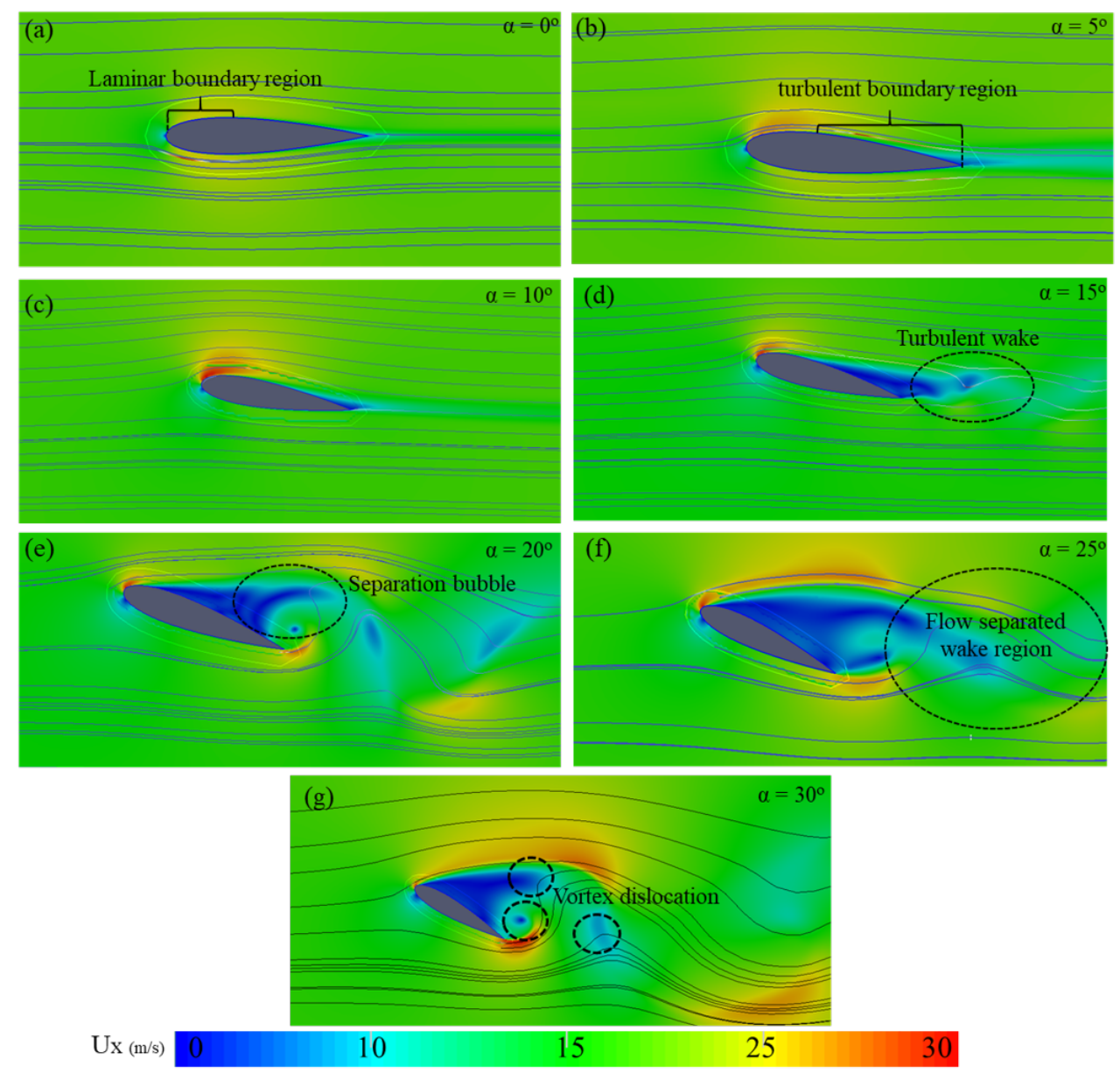

Figure 11. Normalized velocity contours and streamlines for different angle of attack at $\mathrm{Re}=$ $3 \times 10^{5}$ 

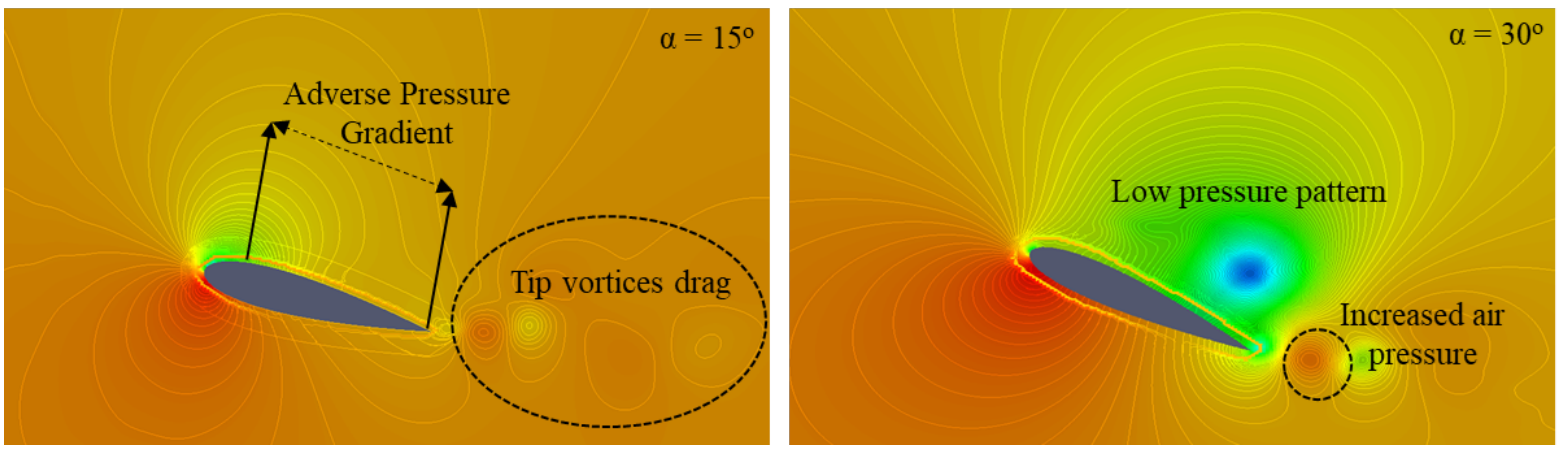

Figure 12. Instantaneous pressure contours at $\mathrm{Re}=3 \times 10^{5}$ for different angle of attack

\section{Conclusion}

The main objective of this work is to validate OpenFOAM new overset mesh capability for finite volume calculations. Flow over an airfoil was discretized using overset mesh and modeled with $k-\varepsilon$ turbulence modeling. The overset functionality of OpenFOAM is able to capture the attached and separated flow field over the airfoil surface and accurately predict the airfoil stall regime above $\alpha=15^{\circ}$. In addition, the trailing edge wake formulation and smallscale vortices are perfectly captured by the overlapping grids. In terms of validation, the aerodynamic coefficients obtained using overset grid are in reasonable agreement with the experimental data. This proves that the new overset mesh utility's cell-to-cell mapping provides opportunity to simulate various complex designs in a single composite domain. As part of future work, we aim to use the overset grid functionality in designing of optimal wind turbine blades.
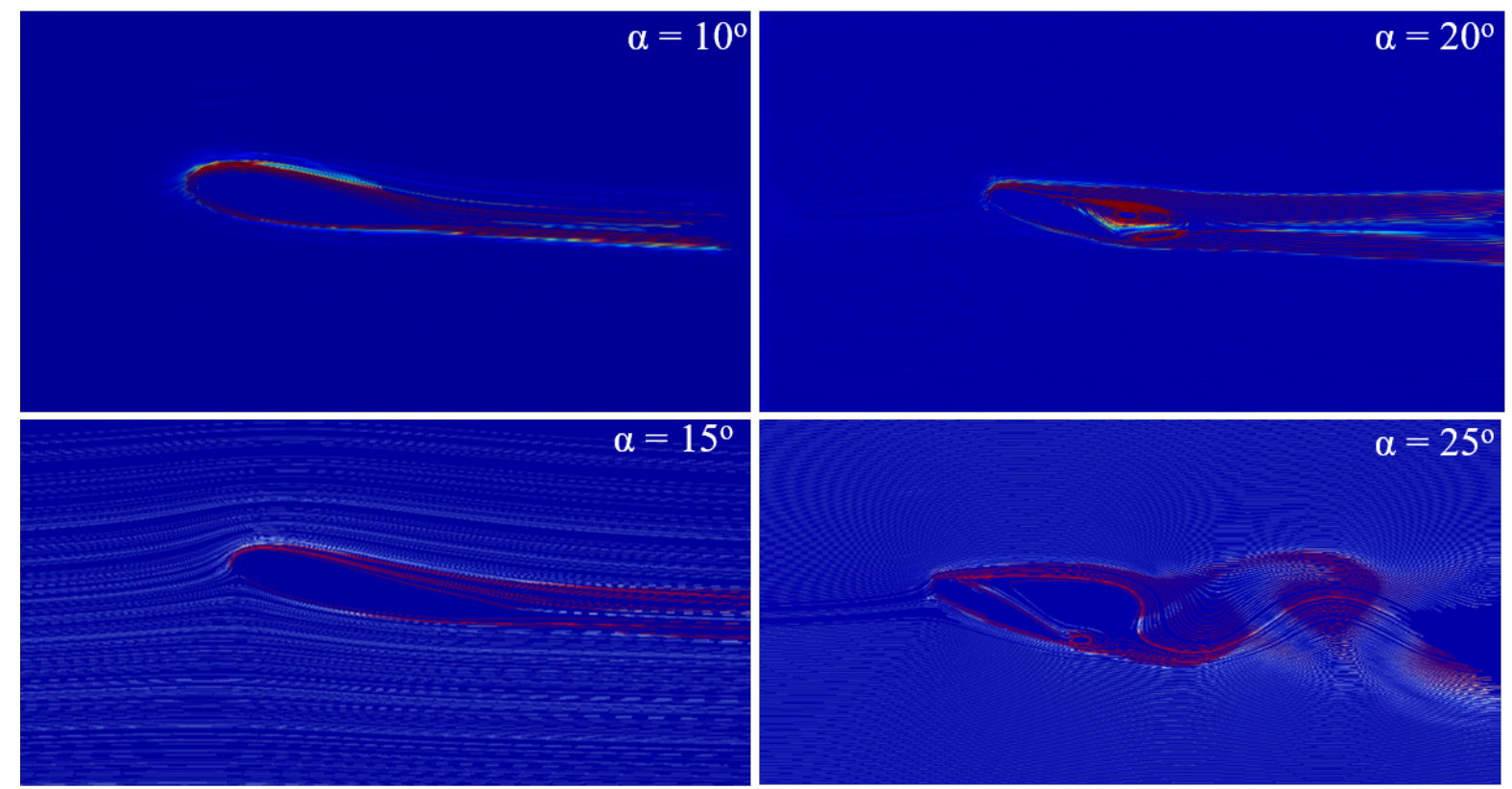

Figure 13. Vorticity streamlines at $\mathrm{Re}=3 \times 10^{5}$ for different angle of attack 


\section{References}

[1] Peskin CS. The immersed boundary method. Acta numerica. 2002; 11:479-517.

[2] Lai MC, Peskin CS. An immersed boundary method with formal second-order accuracy and reduced numerical viscosity. Journal of computational Physics. 2000; 160(2):705-719.

[3] Mittal R, Iaccarino G. Immersed boundary methods. Annu Rev Fluid Mech. 2005; 37:239-261.

[4] Hirt CW, Nichols BD. Volume of uid (VOF) method for the dynamics of free boundaries. Journal of computational physics. 1981; 39(1): 201-225.

[5] Adalsteinsson D, Sethian JA. A fast level set method for propagating interfaces. Journal of computational physics. 1995; 118(2):269-277.

[6] Benek J, Buning P, Steger J. A 3-D chimera grid embedding technique. In: 7th Computational Physics Conference; 1985. p. 1523.

[7] Nirschl H, Dwyer H, Denk V. A Chimera Grid Scheme for the Calculation of the Flow Around Particles in Difficult Geometries. In: Numerical methods for the Navier-Stokes equations. Springer; 1994. p. 191-198.

[8] Guerrero J. Overset composite grids for the simulation of complex moving geometries. DICAT, University of Genoa, Italy. 2006.

[9] Chandar DD, Boppana B, Kumar V. A Comparative Study of Different Overset Grid Solvers Between OpenFOAM, StarCCM+ and Ansys-Fluent. In: 2018 AIAA Aerospace Sciences Meeting; 2018. p. 1564.

[10] Cai J, Tsai HM, Liu F. A parallel viscous ow solver on multi-block overset grids. Computers \& Fluids. 2006; 35(10):1290-1301.

[11] Jarkowski M,Woodgate M, Barakos G, Rokicki J. Towards consistent hybrid overset mesh methods for rotorcraft CFD. International Journal for Numerical Methods in Fluids. 2014; 74(8):543-576.

[12] Timmer W. Two-dimensional low-Reynolds number wind tunnel results for airfoil NACA 0018. Wind engineering. 2008; 32(6):525-537.

[13] Guimet V, Laurence D. A linearised turbulent production in the k- $\varepsilon$ model for engineering applications. In: Engineering Turbulence Modelling and Experiments 5. Elsevier; 2002. p.157-166.

[14] Huang P, Bardina J, Coakley T. Turbulence modeling validation, testing, and development. NASA Technical Memorandum. 1997; 110446.

[15] Mansour N, Kim J, Moin P. Near-wall k-epsilon turbulence modeling. AIAA journal. 1989; 27(8):1068-1073.

[16] Wilcox DC, et al. Turbulence modeling for CFD. vol. 2. DCW industries La Canada, CA; 1998.

[17] Fiabane L, Gohlke M, Cadot O. Characterization of ow contributions to drag and lift of a circular cylinder using a volume expression of the fluid force. European Journal of Mechanics-B/Fluids. 2011; 30(3):311-315.

[18] Roskam J, Lan CTE. Airplane aerodynamics and performance. DARcorporation; 1997.

[19] Launder BE, Spalding DB. The numerical computation of turbulent flows. In: Numerical Prediction of Flow, Heat Transfer, Turbulence and Combustion. Elsevier; 1983. p. 96-116. [20] Liu S. Implementation of a Complete Wall Function for the Standard k epsilon Turbulence Modeling OpenFOAM 4.0. In Proceedings of CFD with OpenSource Software, 2016; 2016. Available from: http://www.tfd.chalmers.se/ hani/kurser/OS_CFD_2016.

[21] Yang Z, Shih TH. A new time scale based K- epsilon model for near wall turbulence. vol. 105768. Lewis Research Center; 1992. 
[22] Geuzaine C, Remacle JF. Gmsh: A 3-D finite element mesh generator with built-in pre and post-processing facilities. International journal for numerical methods in engineering. 2009; 79(11):1309-1331.

[23] Munch C, Ausoni P, Braun O, Farhat M, Avellan F. Fluid \{structure coupling for an oscillating hydrofoil. Journal of Fluids and Structures. 2010; 26(6):1018-1033.

[24] Gopalan H, Jaiman R, Chandar DD. Flow past tandem circular cylinders at high Reynolds numbers using overset grids in OpenFOAM. In: 53rd AIAA Aerospace Sciences Meeting; 2015. p. 0315.

[25] Ferziger JH, Peric M. Computational methods for fluid dynamics. Springer Science \& Business Media; 2012.

[26] Greenshields CJ. OpenFOAM user guide. OpenFOAM Foundation Ltd, version. 2015; $3(1)$.

[27] Ramamurti R, Lohner R. A parallel implicit incompressible ow solver using unstructured meshes. Computers \& Fluids. 1996; 25(2):119-132.

[28] Franke J, Hirsch C, Jensen A, Krus H, Schatzmann M, Westbury P, et al.

Recommendations on the use of CFD in wind engineering. In: Cost action C. vol. 14; 2004. p. C1.

[29] Courant R, Friedrichs K, Lewy H. On the partial difference equations of mathematical physics. IBM journal of Research and Development. 1967; 11(2):215-234.

[30] Weber J. The Calculation of the Pressure Distribution over the Surface of TwoDimensional and Swept Wings with Symmetrical Aerofoil Sections. AERONAUTICAL RESEARCH COUNCIL LONDON (ENGLAND); 1953.

[31] Abbott IH, Von Doenhoff AE. Theory of wing sections, including a summary of airfoil data. Courier Corporation; 1959.

[32] Rusak Z, Morris WJ. Stall Onset on Airfoils at Moderately High Reynolds Number Flows. Journal of Fluids Engineering. 2011; 133(11):111104.

[33] Devinant P, Laverne T, Hureau J. Experimental study of wind-turbine airfoil aerodynamics in high turbulence. Journal of Wind Engineering and Industrial Aerodynamics. 2002; 90(6):689-707.

[34] Seifert A, Darabi A, Wyganski I. Delay of airfoil stall by periodic excitation. Journal of aircraft.1996; 33(4):691-698.

[35] Siddiqui MS, Durrani N, Akhtar I. Quantification of the effects of geometric approximations on the performance of a vertical axis wind turbine. Renewable Energy. 2015; 74:661-670.

[36] Illman PE, Gailey GE. The pilot's handbook of aeronautical knowledge. McGraw-Hill; 2000.

[37] Zhou Y, Alam MM, Yang H, Guo H, Wood D. Fluid forces on a very low Reynolds number airfoil and their prediction. International Journal of Heat and Fluid Flow. 2011; 32(1):329-339.

[38] Nakano T, Fujisawa N, Oguma Y, Takagi Y, Lee S. Experimental study on ow and noise characteristics of NACA0018 airfoil. Journal of Wind Engineering and Industrial Aerodynamics. 2007; 95(7):511-531.

[39] Wang S, Ingham DB, Ma L, Pourkashanian M, Tao Z. Numerical investigations on dynamic stall of low Reynolds number ow around oscillating airfoils. Computers \& Fluids. 2010; 39(9):1529-1541. 